\title{
Le Sénégal à l'heure des médias numériques confessionnels
}

\author{
Le Hizbut-Tarqiyyah et son site Internet, \\ comme reflets de nouveaux lieux de culte virtuels
}

Serigne Sylla ${ }^{1}$

\begin{abstract}
[Résumé] L'évolution des autoroutes de l'information a permis la naissance des médias numériques. Ainsi, Internet et les autres plateformes digitales se sont montrés comme les nouveaux rois de la communication. Dès lors, ils ont modifié les relations sociales et les institutions comme la religion. Ce qui a encouragé l'émergence des médias numériques confessionnels. Cet article les analyse comme de nouveaux lieux de culte en partant du site Internet htcom.sn du groupe Hizbut-Tarqiyyah. Son contenu est étudié en trois points : islam en général, le mouridisme et les faits sociétaux traités selon le point de vue islamique.

Mots-clés: Hizbut-Tarqiyyah, lieu de culte, média numérique confessionnel, mouridisme, vulgarisation.
\end{abstract}

[Abstract] The evolution of information highways has led to digital media. Thus, In-
ternet and others digital platforms showed themselves as the new kings of
communication. From then on, they are upset social relations and institutions like
religion. This encouraged the birth of religious digital media. This article analyzed
them as new places of worship starting from the digital platform htcom.sn of the
group Hizbut-Tariqiyyah. Its content is studied in three parts: Islam in general,
Mouridism and societal facts treated from an Islamic point of view. Keywords: popularization, Hizbut-Tarqiyyah, Muridism, place of worship, religious digital media.

\section{Introduction}

Le Sénégal se caractérise par un contexte religieux singulier avec $96 \%$ de musulmans

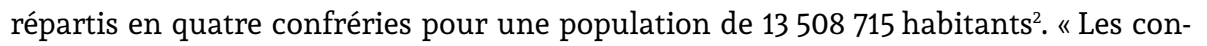
fréries religieuses du Sénégal sont la tidjaniya [Tidianisme] (49\%), la mouridiya [mouridisme] (31 \%), la qadiriya [les Qadrs] (8 \%), et l'ilahiya [les Layènes] (0,6 \%). La tidjaniya et la qadiriya sont d'origines étrangères et portent le nom de leurs fondateurs en occurrence Ahmad Tidjani et Abdel Qadir Al Jilani. La mouridiya et l'ilahiya sont fon-

\footnotetext{
${ }^{1}$ Université Gaston Berger (Sénégal).

${ }^{2}$ Recensement général de la population, de l'habitat, de l'agriculture et de l'élevage, Agence nationale de la statistique et de la démographie, 2013.
} 
dées respectivement par Ahmad Bamba et Limamou Laye, tous deux Sénégalais » (Thiam, 2010 : 37). Parmi ces confréries, les Mourides se distinguent tout particulièrement dans la culture médiatique sénégalaise du fait de leur capacité d'adaptation aux nouvelles technologies (Bèye, 2011) et leur volonté de mise en place de différents canaux de diffusion. Le mouridisme est une confrérie musulmane fondée par le guide religieux sénégalais Cheikh Ahmadou Bamba Mbacké, de son vrai nom Mouhamed Ben Abib Allah (1853-1927), à la fin du XIX siècle. La capitale est Touba, une ville située au centre du Sénégal à $200 \mathrm{~km}$ de Dakar (Guèye, 2002a; Ross, 2006). Des migrants mourides sont aujourd'hui implantés partout dans le monde et ont contribué à la formation d'un réseau confrérique transnational (Bava, 2005).

Le domaine des médias, qui a connu d'importants changements depuis la fin des années 1970 et surtout depuis le début des années 1990, se caractérise au Sénégal notamment par des rivalités entre acteurs politiques, économiques et religieux (Wittmann, 2008). Le paysage médiatique sénégalais est relativement riche du fait de la presse écrite, la radio, la télévision et la presse en ligne. La connexion Internet a officiellement été rendue accessible en 1996 grâce à un travail entrepris à la fin des années 80 par l'ORSTOM (actuel IRD) et l'Enda Tiers Monde'. L'Internet s'est cependant développé avec la SONATEL ${ }^{4}$ et a beaucoup profité à la presse, car " grâce à Internet et à la facilité de ses contacts en situation de mobilité, le paysage audiovisuel sénégalais opère en douceur un changement, avec une offre de sites web qui servent de relais aux grandes chaînes hertziennes dont l'accès n'était pas à la portée de tous » (Bèye, 2011 : 111).

Les mourides se sont aussi saisis de cette évolution des médias, en particulier des médias en ligne, ce qui sera l'objet de cet article focalisé sur les relations entre médias numériques et le mouridisme en partant du cas sénégalais de Hizbut-Tarqiyyah. Hizbut-Tarqiyyah est un dahira (association religieuse) se réclamant du mouridisme. Elle est née en 1975 à l'Université de Dakar sous le nom de Dahira des Étudiants Mourides. Les sites web sont ainsi étudiés comme un prolongement des médias classiques selon Mamadou Ndiaye qui souligne qu'« au Sénégal, loin d'être un handicap pour la presse écrite, l'Internet a été vite compris et perçu comme un nouveau support offrant de nombreuses opportunités. Il a permis aux groupes qui l'ont investi seulement quelques mois après la connexion officielle du Sénégal, survenue en 1996, d'augmenter leur lectorat et de toucher les Sénégalais de la diaspora » (Ndiaye, 2013: 689). Ce phénomène des médias confessionnels des années 2000 mérite en effet d'être considéré à travers différentes figures. Après htcom.sn en 1998, on peut noter le lancement de la radio Lamp Fall Fm en 2002, des chaînes de télévision comme Touba TV en 2010, Lamp Fall TV en 2011... Par la suite, d'autres portails et chaînes en ligne

\footnotetext{
${ }^{3}$ Environnement et développement du tiers monde.

${ }^{4}$ Société nationale des télécommunications.
} 
comme Toubainfos.com, Majalis.org et Bichri TV ont été créés par les mourides aux côtés des médias des autres confessions ou confréries musulmanes comme Mourchid TV et Mourchid FM des Tidianes, Diamalahi FM des Layènes, Espérance FM et Radiomisericordia.info de l'Église catholique. D'ailleurs, le lien entre internet/média et religion est au cœur des problématiques traitées en sciences sociales.

« Du côté du religieux, nous ne pouvons plus ignorer aujourd'hui l'impact d'Internet qui est non seulement un média sur lequel les acteurs religieux agissent et interagissent, mais également un lieu de création et de recréation de la sphère religieuse contemporaine » (Duteil-Ogata et al., 2015: 10). Si Jean-Paul Willaime (2001) souligne le traitement médiatique de la religion et les nombreux biais que cela comporte, DuteilOgata et al. (2015), considérant que la relation entre Internet et religion constitue une problématique toujours d'actualité, se demandant si Internet est une "caisse de résonnance de la modernité religieuse " ou l'" avènement d'une nouvelle modernité " (p. 26). Ces auteurs avancent aussi que la présence du religieux sur la toile « pose donc de nombreuses questions autant aux institutions religieuses, qu'aux croyants ou aux chercheurs en sciences sociales. L'évolution constante des interfaces numériques et de leur fonctionnalité renouvelle sans cesse ces interrogations et laisse de nombreuses questions ouvertes"(Duteil-Ogata et al., 2015: 26-27). En outre, plusieurs études se sont intéressées aux relations entre médias et religions en Afrique de l'Ouest. Frédérick Madore (2016) s'est penché sur l'islam ivoirien et burkinabé à l'ère du web et des réseaux sociaux. Concernant le Sénégal, Cheikhouna Bèye (2011) a interrogé le modèle communicationnel novateur des mourides avec Internet. Julien Bondaz (2015) a pour sa part analysé la rencontre entre la tradition iconographique et les TIC chez les mourides. Cheikh Guèye (2002b) a étudié le rôle des TIC dans les mutations urbaines de Touba, la capitale des mourides et l'appropriation particulière des technologies numériques par les mourides (2003) en partant de l'analyse du site htcom.sn. Ndiaga Loum et Ibrahima Sarr (2017) ont, quant à eux, analysé les rapports de force entre les médias et le pouvoir maraboutique, et les contenus des télévisions d'obédience mouride. Ces diverses études ont ainsi traité des usages et l'appropriation d'Internet par les acteurs religieux, le traitement médiatique partiel de la religion et les rapports de force entre champ médiatique et pouvoir maraboutique. Elles montrent que la question des médias numériques confessionnels considérés comme lieux de culte reste le parent pauvre de la littérature scientifique sur les médias et la religion en Afrique subsaharienne.

Afin de nourrir ce champ peu exploré, cet article vise à comprendre les stratégies de vulgarisation de la culture et de l'identité des mourides mises en œuvre par les fidèles à travers les médias numériques afin d'encourager la pratique des principes de l'islam. Nous avançons que ces derniers sont des lieux de culte virtuels prolongeant ainsi les daara, les dahira et les mosquées tout en œuvrant à la vulgarisation des valeurs et principes du mouridisme. Nous partons du cas de Hizbut-Tarqiyyah et sa plateforme htcom.sn considérée comme un média confessionnel. Par média confession- 
nel, il faut entendre tout média « dont la mission est la diffusion de contenus religieux stricto sensu [...] et d'informations séculières interprétées selon une perspective religieuse [...], sur la totalité du programme » (Chandès, 2013). Et htcom.sn (1998) est justement le premier site web de référence islamique dédié au mouridisme.

Ce travail repose sur des recherches documentaires et empiriques. Des entretiens semi-directifs ont été réalisés avec les responsables de Hizbut-Tariqiyyah et ont été complétés par des séances d'observation directe ainsi qu'une analyse de contenu du site htcom.sn (Sylla, 2017b). Cet article est structuré en deux parties : la première présente Hizbut-Tarqiyyah en tant que pionnier du numérique et rend compte des différentes étapes de son évolution, et la seconde propose une analyse du site internet htcom.sn comme une fenêtre ouverte sur le monde pour mettre en exergue le contenu du mouridisme.

\section{Hizbut-Tarqiyyah : les mutations d'une association religieuse}

Avant de se pencher sur la plateforme Htcom.sn, il importe d'abord de faire un retour sur la naissance et l'évolution de Hizbut-Tarqiyyah ainsi que ses nombreuses réalisations. Pouvant compter sur des moyens financiers considérables, cette association religieuse a fait figure de pionnier du numérique au Sénégal grâce à d’importants investissements.

\subsection{Du dahira à l'organisation religieuse internationale}

Comme l'a expliqué Cheikhouna Bèye (2011), le dahira est « un lieu d'apprentissage de l'enseignement coranique et du comportement d'un bon talibé mouride » (p. 114). Dès lors, Hizbut-Tarqiyyah, qui est né en décembre 1975 à l'Université de Dakar sous le nom de "Dahira des étudiants mourides ", en est un archétype. En effet, après quarante ans d'existence, il s'agit aujourd'hui d'une organisation mouride présente dans les quatorze régions du Sénégal et à l'étranger : en Afrique, en Europe, dans les 50 états des États-Unis, au Canada, en Amérique du Sud et en Asie. En 1992, le dahira est baptisé Hizbut-Tarqiyyah. Ce nom, donné par le Khalife des mourides d'alors, Serigne Saliou Mbacké, signifie «la faction des gens dont l'ascension spirituelle auprès de DIEU se fait par la Grâce et directement sous les auspices de leur maître, le Serviteur du Prophète Khadimou Rassoul ॥. L'année 1992 marque aussi le début de la construction du siège et du complexe culturel de l'institution. Il est situé à Touba et couvre une superficie de 7 ha. Pour piloter ses activités, Hizbut-Tarqiyyah a mis en place un organigramme reposant sur une administration générale qui coordonne avec ses diffé-

${ }^{5}$ Consulté le 10 décembre 2017, URL : www.htcom.sn. 
rentes cellules les programmes et les objectifs de l'organisation. Ainsi, ce mouvement compte de nombreuses instances et structures spécialisées: "Administration et Finances », "Enseignement et Éducation »; "Culture et Civilisation»; «Études, Recherches et Documentation»; «Social»; «Coopération»; «Technologie de l'Information et de la Communication »; "Activités et formation professionnelle »; «Activités économiques d'entraide et de promotion»; «Agriculture et élevage». Ses domaines d'activité sont orientés autour de l'enseignement et l'éducation, la formation religieuse, l'initiation au travail et la vulgarisation de l'œuvre et de la mission du Cheikh Ahmadou Bamba, le guide spirituel et fondateur du mouridisme.

Hizbut-Tarqiyyah compte à son actif de nombreuses réalisations : le groupe scolaire Daroul Alimil Khabir présent à Touba, Dakar et Kaolack avec les cycles du préscolaire, de l'élémentaire et du moyen secondaire ; un Institut international d'études et de recherches sur le mouridisme (IIERM), qui reçoit des chercheurs venant de différents endroits du monde et qui organise les grandes expositions sur le mouridisme chaque année à l'occasion du Grand Magal de Touba ${ }^{6}$; des espaces culturels pour la formation des adolescents, des hommes et des femmes; une mosquée pour l'office de la prière et l'enseignement des sciences fondamentales islamiques ; un atelier de formation professionnelle en menuiserie métallique, en menuiserie bois, en électricité et en génie civil ; ainsi que des exploitations agricoles dans la vallée du fleuve Sénégal pour la prise en charge des besoins de l'entité pendant la période du Magal.

En outre, il faut noter que ce dahira a été créé dans le but de promouvoir les valeurs culturelles et identitaires du mouridisme. C'est un centre culturel animé par deux principales motivations selon El hadj Ibrahima Thiam (2010) : "D’une part, le désir ardent de retrouver la véritable personnalité musulmane et l'identité culturelle et d'autre part le souhait de réhabiliter le patrimoine culturel et religieux de l'islam prôné par Cheikh Ahmadou Bamba»(p.103). Les disciples mourides se réclamant de Hizbut-Tarqiyyah sont reconnus aussi par leur tenue vestimentaire qu'on appelle les Baye Lahad du nom du troisième Khalife des Mourides, Serigne Abdoul Ahad (19681989). Celui-ci « s'habillait d'un grand boubou aux manches très larges, un bonnet sur la tête et autour du cou une longue écharpe faite du même tissu, le tout étant accompagné d'une pochette en cuir pendue jusqu'à hauteur de la poitrine» (Thiam, 2010 : 107).

Par ailleurs, Modou Diome (2012) fait la corrélation entre la naissance de HizbutTarqiyyah et l'insatisfaction d'une jeunesse à l'égard des doctrines marxistes et socialistes dans les années 1970 et 1980. À propos de leur guide et inspirateur, Serigne Abdoul Ahad, Baye Lahad note que :

\footnotetext{
${ }^{6}$ Cet évènement est célébré chaque année à Touba, la capitale des mourides. II marque la commémoration du départ en exil du Cheikh Ahmadou Bamba (fondateur de la confrérie) au Gabon de 1895 à 1902.
} 
Par sa posture tribunitienne, Baye Lahat polarise l'attention et l'affection des jeunes élèves et étudiants mourides qui désormais, s'identifient à lui dans la pensée, l'idéologie, le mode d'habillement et l'engagement. Ils se retrouvent dans les discours tranchants du Khalife général des mourides et tiennent la dragée haute à un État jugé prédateur et suppôt de l'Occident (Diome, 2012 : 60).

Dans la même veine, le 15 avril 1995, lors de la célébration du centenaire du départ en exil de Cheikh Ahmadou Bamba célébré par Hizbut-Tarqiyyah à Dakar, son leader, Atou Diagne avait déclaré :

Si tu es un président de la République ou une puissance étrangère, sache que, cet héritage [culturel du mouridisme] et ce Magal, ont plus de valeur que ce, avec quoi tu veux nous corrompre. Nous n'en voulons pas! Si tu es une richissime personnalité qui sponsorisait d'autres événements, retournes y, Serigne Touba s'est révélé en de plus jeunes gens [comme nous]. Accroche-toi pour qu'ils te remorquent.

Pour ce qui est des sources de son financement, qui a lui a permis d'assurer son fonctionnement, de permettre ses réalisations et de réaliser d'importants investissements sur les plans social, économique et technique, Hizbut-Tarqiyyah s'est toujours appuyé sur les cotisations de ses membres. En effet, si l'on en croit un dossier réalisé par xibar.net, " déjà en 1995, trois mois avant la célébration du centenaire du départ en exil de Cheikh Ahmadou Bamba, le Hizbut Tarqiyyah avait, selon des sources sûres, mobilisé un budget de 600 millions de francs CFA, provenant essentiellement des cotisations mensuelles de ses membres ${ }^{7}$ ». Ceci était d'autant plus impressionnant qu’à cette période, il ne comptait que 10000 membres, selon ce dossier. À noter surtout en ce qui nous concerne que d'importants investissements financiers dans le numérique ont fait de Hizbut-Tarqiyyah un des pionniers des médias numériques au Sénégal.

\subsection{Une référence dans le numérique au Sénégal}

L'organisation est promotrice de supports médiatiques importants qui vont d'Internet à la télévision numérique en passant par la radio. Les investissements dans ce secteur ont débuté à la fin des années 1990 avec htcom.sn (1998). Cheikh Guèye explique à ce sujet qu' « à la fin des années 80 , alors que l'informatique était à ses débuts au Sénégal, le dahira s'est doté d'une flotte d'ordinateurs qui a suscité l'émerveillement et l'envie dans plusieurs milieux dakarois. Cette acquisition avait pour but de s'adapter à la mutation du dahira dont la mobilité des membres nécessitait un contrôle plus serré à distance » (Guèye, 2002b : 28). Mais force est de constater par ailleurs que cette informa-

\footnotetext{
${ }^{7}$ Consulté le 10 décembre 2017, URL: http://www.xibar.net/dossier-a-la-decouverte-duhizbut-tarqiyyah-le-bras-technique-des-khalifes-de-la-mouridiya_a7795.html.
} 
tisation du dahira renferme une certaine signification aux yeux de ses membres. En effet, toujours selon Cheikh Guèye, «l'informatisation était également pour Hizbut Tarqiyya un moyen de conformer son fonctionnement à l'image de puissance de Cheikh Ahmadou Bamba. Il s'agissait aussi de former les membres à cet outil qui était déjà considéré comme une véritable révolution » (Guèye, 2002b : 29).

Ce point de vue la formation des membres à l'informatique ont fait de HizbutTarqiyyah le grand géant du numérique qu'il est aujourd'hui. Dès lors, le site web Orbit Informatique suptic soulignait « le rôle que ses milliers de membres jouent dans le domaine de la communication avec l'instauration d'une radio Touba HizbutTarqiyyah 95.6 FM [2013], la télévision Al Mouridiyyah TV, un des pionniers de l'IPTV en Afrique" ». Selon le président d'Al Mouridiyyah TV, Atou Diagne : «Al Mouridiyyah $T V$ est un outil qui vient compléter la gamme des solutions sur les TIC dans lesquelles Hizbut-Tarqiyyah s'est toujours investie pour vulgariser les enseignements de Cheikh Ahmadou Bamba : un premier serveur Videotex en 1988, une radio diffusion en 1995, le premier portail web de référence sur le mouridisme (htcom.sn) en $1998 . .{ }^{10} »$. Al Mourdiyyah TV n'est donc que l'aboutissement d'un long processus de création d'un média de vulgarisation du mouridisme.

Cela dit, pour comprendre l'émergence de Hizbut-Tarqiyyah et des médias d'obédience mouride, il reste important de rappeler le différend qui avait opposé la confrérie mouride à l'ORTS (actuelle RTS ${ }^{11}$ ) en 1983. C'est à la suite d'un refus de l'ORTS de diffuser une déclaration de Serigne Abdoul Ahad, alors Khalife Général des mourides, que celui-ci était intervenu pour exhorter les taalibés mourides à boycotter ce média public à la faveur des moyens traditionnels de communication typiquement mourides. Il avait alors déclaré : «Si la [RTS] n'est pas capable de couvrir entièrement les évènements de la confrérie au même titre que les autres évènements, qu'on le considère comme la radio qui est au Mali. À partir d'aujourd'hui, poursuit-il, tout ce qui se passera dans la confrérie sera diffusé au niveau de la radio [les mégaphones] de la grande mosquée de Touba [...] ». Ce message avait fortement encouragé les mourides à monter leur propre groupe de communication et peut être considéré comme un déclencheur de la prolifération des médias confessionnels mourides au Sénégal (Sylla, 2015).

Hizbut-Tarqiyyah est par conséquent cité parmi les initiateurs des médias religieux au Sénégal (Diome, 2012). En effet, son engagement à satisfaire les besoins de la communauté mouride dans le domaine de l'information et de la communication a connu

\footnotetext{
${ }^{8}$ Cette radio porte aujourd'hui le nom d'Al Mouridiyyah FM.

${ }^{9}$ Consulté le 10 décembre 2017, URL : www.orbiti.com/hizbut-tarqiyyah-a-la-decouvertedun-grand-acteur-de-developpement.

${ }^{10}$ Enquêtes de terrain - thèse: Atou Diagne, le président de Hizbut-Tarqiyyah, discours d'inauguration d'Al Mouridiyyah TV, Touba, décembre 2013.

${ }^{11}$ Radiodiffusion télévision sénégalaise.
} 
une première expérience avortée avec la radio Touba FM, lancée sans autorisation en 1995 lors du Magal de Touba de la même année. Ceci constituait alors une forme de défiance de Hizbut-Tarqiyyah vis-à-vis de l'État :

Le désir de création de leur propre radio se justifie par le fait que cette communauté ne s'est jamais reconnue dans les programmes offerts par les médias d'État. Il leur fallait une parade. Elle s'est posée à la fois avec effet d'annonce et effet de surprise, démontrant par-là, leur farouche détermination à combattre par tous les moyens, la laïcité et son corolaire: l'occidentalisation (Diome, $2012: 60)$.

Ce projet a été réalisé via le site web htcom.sn que nous considérons comme une fenêtre ouverte sur le monde pour mettre en exergue le contenu du mouridisme en raison de son orientation confessionnelle.

\section{Htcom.sn : la fenêtre ouverte du mouridisme sur le monde}

Htcom.sn est un site dédié au mouridisme et à son fondateur. Sa création en 1998 marque une évolution du dahira qui se tourne dès lors vers le numérique. C'est à ce titre que Guèye affirme que " the creation of a website (www.htcom.sn) is a new stage in the evolution of the dahira and demonstrates its avant-garde awareness of, and interest in, utilising NICTs to further Mouride universalism. Although still under construction, the site demonstrates a high degree of awareness within the movement of the new challenges posed by NICTs» (2003: 622). Dans cette partie, l'accent sera mis, d'une part, sur l'étude de la référence religieuse et confrérique de la ligne éditoriale de htcom.sn et, d'autre part, sur le fait qu'il soit, selon notre analyse, un lieu de culte virtuel prolongeant les daara, les dahira et les mosquées.

\subsection{Le mouridisme et son fondateur au centre du site Internet}

La référence au mouridisme de htcom.sn peut être observée selon trois éléments. Premièrement, celle-ci est définie d'entrée de jeu à travers son lien de direction, « Khâdimu-r-Rasûl, Le Serviteur privilégié du Prophète (PSL) ». D’emblée le visiteur comprend qu'il se dirige vers une page dédiée au mouridisme. En outre, en se présentant comme le «Serviteur privilégié » du prophète, Hizbut-Tarqiyyah place le Cheikh au rang des savants de l'Islam.

Deuxièmement, les images qui font le décor du site réfèrent toutes au mouridisme. À droite de la page d'accueil (voir photo) une photo de Cheikh Ahmadou Bamba est mise à côté de celle de la mosquée de Touba et le premier verset du Coran écrit en 
$\operatorname{arabe}^{12}$. À gauche se trouve une photo de l'actuel Khalife des mourides, Cheikh Sidy Moukhtar Mbacké ${ }^{13}$. Au milieu, est montré en filigrane la représentation du Grand Magal de Touba qui est célébré chaque année. Ce type de communication fondé sur les signes distinctifs et les images, est une stratégie que les mourides partagent avec les mouvements pentecôtistes contemporains africains et de l'Amérique latine, dont le prosélytisme se fonde en grande partie sur l'usage des multimédias. Plus précisément, il s'agit d'une communication basée sur le charisme et la personnalité d'un lea$\operatorname{der}$ (Asamoah-Gyadu, 2014).

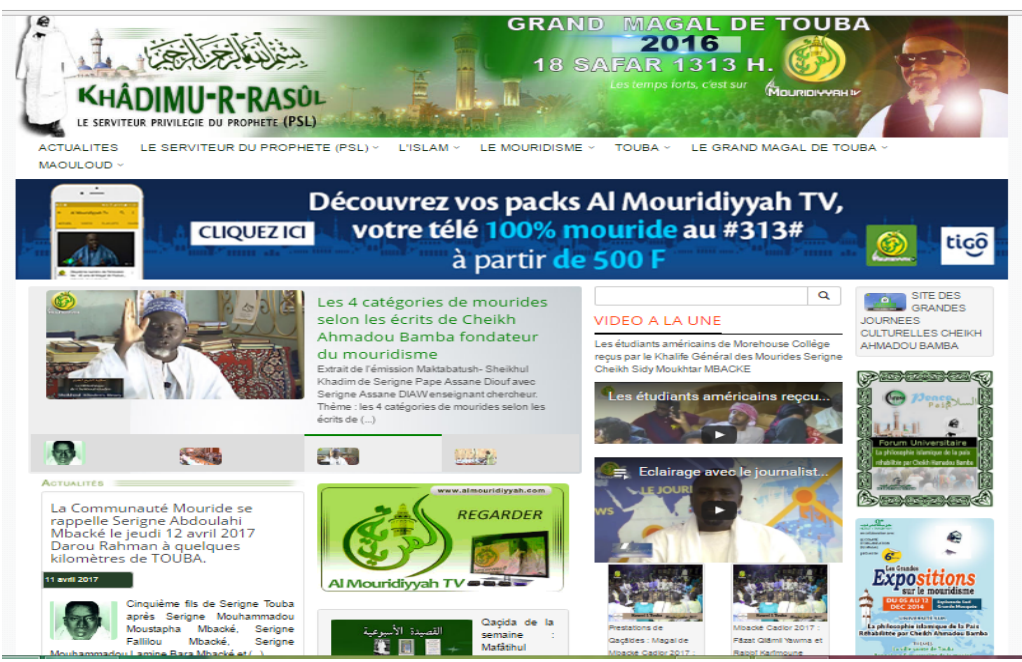

Photo : capture d'écran de la page d'accueil du site www.htcom.sn (consulté le 10 décembre 2017)

Le mouridisme est ainsi présenté sous ses aspects culturels avec son khalifat, sa mosquée, sa capitale, ses fidèles et ses grands évènements. C'est ainsi une manifestation d'un «désir de maintien et d'affirmation identitaire ethnoculturelle et ethnoreligieuse » (Marcotte, 2016 : 10). La représentation de la confrérie mouride par les images (Sylla, 2017a) constitue une stratégie de promotion des valeurs culturelles et identitaires des taalibé [disciples] mourides sur la toile. Elle permet aussi de toucher un immense public dans ou hors du territoire sénégalais. C'est une stratégie de communication religieuse utilisée aujourd'hui par toutes les confessions conscientes de l'importance du numérique. Parmi les usagers, il est important de noter les mouvements pentecôtistes africains qui cherchent toujours à rester en contact avec leur diaspora (Hackett, 2009). C'est d'ailleurs dans cette même perspective que Guildas

\footnotetext{
${ }^{12}$ Bismil lahi rahmanir rahim (Au nom de Dieu, Clément et Miséricorde, le Très Miséricordieux).

${ }^{13}$ Petit-fils du fondateur de la confrérie et septième khalife depuis 2010.
} 
Mouthé qui, dans son analyse des relations entre le catholicisme et les usages religieux d'internet au Cameroun, souligne que « l'Église catholique considère l'internet comme un instrument indispensable pour la mission d'évangélisation. Le Saint-Siège indique l'importance des nouveaux médias pour la diffusion de l'évangile » (Mouthé, 2016:10-11). De telles pratiques ont sans doute fait naître des concepts comme « cyber pastorale », « cyber rituels », « cyber commerce »... (Mouthé, 2016) mobilisés par des analystes pour rendre compte des implications des médias sociaux dans la vie religieuse.

Enfin, le curseur est mis sur deux principaux domaines que sont les publicités et les informations (images, vidéos et textes présents sur le site). Ce qu'il nous semble important de noter est que les publicités affichées sur htcom.sn sont toujours sélectionnées selon des critères correspondant à l'islam. Ainsi, la plupart tournent autour des évènements religieux mourides comme des Magal, des Gamou... À côté de ces annonces se trouvent aussi d'autres publicités qui, même si elles ne sont pas religieuses, restent acceptées par les principes de l'islam tels que les opérateurs de téléphonie mobile et les accessoires électroniques, car ils ne sortent pas des principes du halal ou du travail licite. Or, pour les mourides, le fait de se conformer dans le cadre travail au halal est le fruit d'une recommandation du Cheikh Ahmadou Bamba, qui utilisait dans ses écrits «les expressions arabo-musulmanes talab-ul-halâl, kasb-ul-halâl ou itlimâsul-halâl dont la traduction littérale est chercher le licite » (Ndiaye, 2010 : 171). De plus, les informations sur l'actualité portent souvent sur des thèmes ayant trait à l'islam et plus spécifiquement au mouridisme. Le reste des informations est donné sous forme de dossiers et de reportages traitant des grands sites, des œuvres et des grands évènements du mouridisme. C'est la raison pour laquelle la publicité de produits contraires aux principes de l'islam et les informations inhérentes aux faits divers ou aux choses folkloriques comme le sport ou la musique sont totalement absentes sur htcom.sn.

La mission d'éducation religieuse que joue ce site internet est plus visible sur les pages « Actualités », « Le Serviteur du Prophète (PSL) », « L'Islam », « Le Mouridisme », "Touba », «Le Grand Magal de Touba » et « Maouloud » du site. Par exemple, la page «Le Serviteur du Prophète (PSL) » est dédiée exclusivement à Ahmadou Bamba, à sa vie, son œuvre et sa mission en tant que Cheikh et son statut de «serviteur privilégié du Prophète Mohamed (PSL) ». Pour ce qui est de celle intitulée « Islam », elle affiche un tableau des valeurs de base du mouridisme présenté en cinq points (l'hagiographie du mouridisme, les valeurs historiques du mouridisme, les valeurs littéraires, les valeurs scientifiques et morales et les valeurs artistiques). Elle est prolongée dans la page « Le Mouridisme » avec la définition de la confrérie, son historique, son Khalifat, son expansion et son développement, la famille du Cheikh ainsi que ses grands compagnons. La page "Touba » présente quant à elle la capitale du mouridisme comme un grand pôle urbain. Partant de sa Grande Mosquée, Touba est expliquée dans son historique, son organisation sociale, son organisation administrative et son statut de 
ville sainte. La page «Grand Magal de Touba » est pour sa part dédiée à l'évènement du même nom en expliquant ses fondements, ses significations, l'action de grâce à l'occasion du Magal et les grâces attachées à ce jour. La dernière page "Maouloud» est présentée de la même manière avec son sens et sa portée, les enseignements du Cheikh sur l'évènement, sa considération par le Cheikh, ses recueils de panégyriques sur le prophète Mohamed, les rappels et les exhortations des Khalifes et des reportages sur l'évènement.

$\mathrm{Au}$ travers de cette description, nous voyons à quel point htcom.sn est une vitrine ouverte sur le mouridisme, un canal de vulgarisation de ses valeurs et ses principes. C'est sans doute une des raisons pour lesquelles Jean Copans avait écrit que «Touba est branchée sur le Sénégal, mais c'est l'international qui est branché sur Touba » (Copans cité par Bondaz, 2015 : 271). Le caractère hétérogène et complexe, « la nature ambiguë, insaisissable, presque volatile de l'internet, ainsi que son caractère composite » (Hackett, 2005 : 87) explique toute son importance dans ce contexte.

\subsection{Un site Internet comme lieu de culte virtuel}

Les deux Un lieu de culte est un espace servant à des fidèles pour réaliser divers rituels, magnifier leur croyance, et un lieu d'enseignement de la religion. L'analyse des thématiques des sections "Le Moniteur », «Didacticiel » et « Heures de prières " présentes sur le site htcom.sn montre qu'il est possible de considérer cette plateforme comme un lieu de culte virtuel.

Tout le contenu de la rubrique « Le Moniteur » va dans le sens de la connaissance et de la pratique de l'islam, ce qui fait de htcom.sn un « daara virtuel ». Le daara ou école coranique est construit pour faciliter la formation des fidèles sur le plan de la connaissance et de la maîtrise du Coran (Sylla, 2015). Lamine Ndiaye (2010) parle ainsi de ces daara-jaang (écoles coraniques) pour montrer la place de l'éducation religieuse islamique dans la doctrine du mouridisme. Il les différencie surtout des daara-ligéey ou daara-tarbiya, qui sont des «centres de formation au monde l'ésotérique et aux métiers les plus divers » (Ndiaye, $2010: 174)$. Ainsi, le premier point « Savoir lire rapidement », ayant pour objectif l'enseignement de l'alphabet arabe - qui est la langue de l'islam -, peut être assimilé à ces daara-jaang. Il est prolongé dans le deuxième point, "Initiation au Saint Coran », où sont enseignés le Coran et son exégèse avec des techniques qui ont pour objectif de permettre de lire et de comprendre rapidement les versets. Cet objectif est aussi poursuivi dans «Exercices, études et révisions sur les sciences religieuses » qui enseigne les sciences religieuses. Dans « Usages et rites bon à connaitre », htcom.sn se lance dans un exercice de séparation du permis (le halal) et de l'interdit (le haram) dans la religion. La section, "Coran: commentaires et chroniques ", livre des explications et des traductions du Coran en français, "Hadiths", 
«Ouvrage et législation Malékite » et des enseignements de la pratique de l'islam selon le point de vue de l'école d'Imam Malik ${ }^{14}$. Enfin, "Ã l'école du mouridisme » explique les principes de base de la confrérie. Htcom.sn peut être donc considéré comme un prolongement des daara. En effet, tout son contenu est orienté vers l'enseignement du Coran et des pratiques de l'islam. Dès lors, Bèye insiste la capacité d'adaptation des mourides qui ont su saisir les opportunités offertes par Internet pour améliorer leurs manières de faire religieuses : « Ces expositions virtuelles témoignent à quel point les mourides prennent au sérieux les nouvelles possibilités de communication et le caractère novateur de l'internet $»$ (Bèye, 2011 : 117).

La section «Didacticiel » est une rubrique en quatre points. «Sciences religieuses » explique la théologie, la jurisprudence, le perfectionnement spirituel, la politesse légale... «Panégyriques du Prophète (PSL) » expose les chansons des poèmes (khassida) du Cheikh sur le prophète Mohamed et leurs explications. "Édifications et Sagesse » livre ses paroles sur la religion et la vie sociale rapportées par ses contemporains. "Oraisons initiatiques et incantatoires " présente un certain nombre de rituels que peut faire le fidèle pour améliorer sa croyance et recevoir des récompenses divines. Cette rubrique est un prolongement des dahira, qui sont des associations religieuses réunies autour d'un guide spirituel, dont le but est de chanter les panégyriques et d'enseigner les sciences religieuses. Ils se spécialisent dans le domaine de la déclamation des écrits du Cheikh et de la vulgarisation de son enseignement tant au niveau scientifique que culturel (Sylla, 2015). Momar Coumba Diop explique que «le dahira constitue une structure d'encadrement des taalibe [disciples], un maillon essentiel dans les circuits de communication entre administration confrérique et la masse des fidèles » (Diop, 1981: 80). Il ajoute même que « la propagande est une des activités les plus manifestes des dahira » (Diop, 1981: 80). Par ailleurs, Cheikh Guèye prend les TIC pour des instruments pouvant créer des formes d'identité et de sociabilité dans un contexte de dispersion des individus d'un même groupe social comme la confrérie: «Les NTIC contribuent à la création d'autres formes d'identités et de sociabilités, se nourrissent ou s'accommodent de la distance et réduisent le prosélytisme mouride plus efficace, le conformant ainsi à ses ambitions universalistes » (Guèye, 2002b :172). Ainsi, le portail htcom.sn, étant une fenêtre ouverte sur le monde pour mettre en exergue le contenu du mouridisme, joue un rôle de reliance entre des fidèles séparés par une longue distance.

Enfin, htcom.sn, en rappelant les cinq heures de prière quotidiennes obligatoires de l'islam selon les mois du calendrier grégorien sur la page « Heures de prières ", peut dès lors être vu comme une mosquée virtuelle dans la mesure où il fait le rappel des heures de prière Tous ces éléments montrent à quel point la vie religieuse a et est gérée à proprement parler une place sur le web, et montre, plus que la pertinence, la né-

\footnotetext{
${ }^{14}$ Une des quatre écoles classiques du droit musulman sunnite.
} 
cessité d'une communication religieuse adaptée au numérique. Comme l'avait observé Madore (2016) pour certains musulmans en Côte d'Ivoire et au Burkina Faso, de nouvelles formes de religiosité sont apparues grâce au web dans le cas des Mourides au Sénégal.

Ce portail est en effet un lieu de culte virtuel. Il se présente premièrement comme un prolongement des daara par l'enseignement coranique et religieux qu'il offre. Ici, il s'intéresse à l'islam en général pour expliquer ses principes et ses pratiques. Deuxièmement, htcom.sn joue le rôle, ou du moins fait figure de dahira par la déclamation et l'explication des chansons (khassida) de Cheikh Ahmadou Bamba sur le prophète Mahomet. À cet effet, il se montre très «sectariste" en se limitant exclusivement à la confrérie mouride et principalement à ses aspects culturels. Troisièmement, en s'intéressant aux heures de prières, htcom.sn se substitue à une mosquée. Nous pouvons donc aisément en conclure que, « dès lors que les médias religieux se donnent comme objectif de se substituer ou de compléter les lieux de culte, le média devient un nouveau lieu de culte », écrit Alymana Bathily (2009: 113).

Ces relations entre le portail numérique htcom.sn et ces lieux de culte peuvent être expliquées par la théorie de «l'équivalent fonctionnel » de Robert King Merton (2006). Il a émis cette théorie pour expliquer qu'au sein d'une même société, deux institutions peuvent coexister et remplir la même fonction. Selon lui, une même fonction puisse être remplie par plusieurs organismes qui peuvent être alors complémentaires, mais aussi en concurrence ou en substitution. Or, notre analyse démontre une complémentarité entre htcom.sn et ces institutions. Cependant, l'innovation qu'introduit le numérique, qui en favorisant une neutralisation des espaces (Mucchiellli, 2001), permet le travail à distance, est remarquable. Ceci donne l'opportunité à tout un chacun, quelle que soit la distance qui le sépare de ces lieux de culte, de pouvoir se rattraper grâce à un simple clic. Bertrand Ouellet (2007) montre l'impact de la révolution numérique sur la religion et affirme que «la génération du zap et du clic saura bien, comme toutes les générations, vivre et dire son expérience de vie et son expérience de foi. Le défi, pour nous, croyants, chrétiens, catholiques, [...] notre défi est le défi de la parole : comment dire l'expérience de foi qui est la nôtre pour qu'elle soit comprise et féconde pour la génération qui nous suit» (Ouellet, 2007 : 12). Partant de cela, il est donc fondamental de regarder les portails numériques tels que htcom.sn comme un reflet, voire un prolongement des lieux de culte.

\section{Conclusion}

L'objectif de cet article était de décrire les relations entre le mouridisme et le numérique en partant du cas du dahira Hizbut-Tarqiyyah et de son portail htcom.sn ouvert depuis 1998. La pertinence de cet article portant sur le nouveau visage du paysage médiatique sénégalais tient dans les profondes mutations de la communication mass médiatique. En effet, les médias ont suivi une évolution très rapide qui a fait naître la 
société de l'information et le tout communicationnel. Ce phénomène a ainsi bouleversé les relations sociales et les institutions comme la religion, dont les acteurs ont vite compris l'importance des outils de diffusion de l'information pour la promotion du sacré. Ainsi sont nés les médias confessionnels sous différentes formes telles que le numérique, médias numériques confessionnels que cet article propose de penser comme nouveaux lieux de culte, et le cas de Hizbut-Tarqiyyah avec htcom.sn en est une parfaite illustration.

Ce site se veut une fenêtre ouverte sur le monde pour mettre en exergue le contenu du mouridisme. Il est de ce fait assimilé à un daara virtuel au sens d'école coranique, à une mosquée virtuelle et à un dahira virtuel, car par son contenu, le portail htcom.sn aide réellement à la connaissance et à la pratique de l'islam et une meilleure compréhension des principes du mouridisme. De ce fait, le numérique fait office d'outil servant le prosélytisme religieux dans ce contexte de sécularisation. Par conséquent, au Sénégal comme en Afrique subsaharienne, les sites web se posent dès lors comme des outils de vulgarisation et d'enseignement de la religion sous une nouvelle forme. Bien plus, l'investissement de Hizbut-Tarqiyyah dans le domaine du numérique ne se limite pas seulement aux sites web. En effet, ce groupe gère aussi la radio Al Mouridiyyah FM depuis 2013, et sa réalisation la plus importante dans ce domaine est IPTV Al Mouridiyyah TV. Lancée elle aussi en 2013, elle a constitué une première dans l'histoire des médias au Sénégal et en Afrique subsaharienne. Alors qu'Hizbut-Tarqiyyah vient de fêter ses 40 ans, elle est aujourd'hui citée par les géants du numérique et de l'IPTV sur l'ensemble du continent africain, et continuera donc sans conteste de faire vivre les relations entre religion et médias numérique.

\section{Bibliographie}

ASAMOAH-GYADU J. K. (2014), " "We Are on the Internet”: Contemporary Pentecostalism in Africa and the New Culture of Online Religion », in R. I. J. HACKETT, B. F. SOARES (dir.), New Media and Religious Transformations in Africa, Bloomington, Indiana University Press, p. 157-170.

Bathily A. (2009), Médias et religions en Afrique occidentale, Dakar, Institut PANOS/Afrique de l'Ouest.

BAVA S. (2005), « Le dahira, lieu de pouvoir et d'émergence de nouvelles élites au sein du mouridisme », in M. Gomez-Perez (dir.), L’islam politique au sud du Sahara : identités, discours et enjeux, Paris, Karthala, p. 159-175.

BÈYE C. (2011), «Les mourides du Sénégal face aux défis des nouvelles technologies, le cas d'Internet », Le Temps des médias, vol. 17, n² 2, p. 111-117.

BONDAZ J. (2015), « Images cultuelles et écrans mourides, deux cas de controverse iconographique et religieuse sur Internet », in F. DUtEIL-OGATA, I. JONVEAUX, 
L. KUCKZYNSKI, S. NiZARD (dir.), Le religieux sur Internet, Paris, L'Harmattan, p. 261274.

CHANDÈs G. (2013), « Stations de radio confessionnelles : leur champ de présence sonore », Communication, vol. 31, $\mathrm{n}^{\circ}$ 1, p. 1-9.

Diome M. (2012), Média, mouvement populaire et violence politique : le contrat social sénégalais à l'épreuve de la contestation et des urnes (2000-2012), thèse de doctorat de Sociologie, Saint-Louis, Université Gaston Berger.

Diop M.-C. (1981), « Fonctions et activités des dahira mourides urbains (Sénégal) », Cahiers d'études africaines, vol. 21, n 81-83, p. 79-91.

DuteIL-Ogata F., JonVEAUX I., KuCZYNSKi L., NiZARD, S. (2015), « Introduction. Le religieux sur Internet : textes et contextes », in DuteIL-Ogata F., JonveAux I., KUCZYNSKi L., NiZARD, S. (dir.), Le religieux sur Internet, Paris, L’Harmattan, p. 9-27.

GuÈYE C. (2002a), Touba. La capitale des Mourides, Paris, Karthala.

GuĖYE C. (2002b), « Enjeux et rôle des TIC dans les mutations urbaines : le cas de Touba », in M.-C. Diop (dir.), Le Sénégal à l'heure de l'information : technologies et sociétés, Paris, Karthala.

GuÈYE C. (2003), « New Information \& Communication Technology Use by Muslim Mourides in Senegal », Review of African Political Economy, vol. 30, $\mathrm{n}^{\circ}$ 98, p. 609625 .

HACKETT R. I. J. (2005), « Religion et Internet », Diogène, vol. 211, n 3, p. 86-99.

Hackett R. I. J. (2009), «The New Virtual (Inter)Face of African Pentecostalism», Society, vol. 46, n6, p. 496-503

LOUM N., SARR I. (2017), « Les médias et la confrérie mouride au Sénégal », Revue Internationale des Francophonies, $\mathrm{n}^{\circ}$ 9. En ligne, consulté le 10 décembre 2017. URL : http://rifrancophonies.com/rif/index.php?id=374.

MADORE F. (2016), « L'islam ivoirien et burkinabé à l'ère du numérique 2.0 », Journal des anthropologues, $\mathrm{n}^{\circ}$ 146-147, p. 151-178.

Marcotte R. D. (2016), « Présentation. Perspectives sur les nouvelles modalités des croyances et pratiques religieuses migrantes », Religiologiques, $n^{\circ} 33$, p. 5-27.

Merton R. K. (2006), Éléments de théorie et de méthode sociologique, Paris, Armand Colin.

Mouthe G. (2016), « Catholicisme et usages religieux de l’internet au Cameroun », ticessociété « Les religions au temps du numérique », vol. 9, n 1-2.

MucChIElli A. (2001), Les Sciences de l'information et de la communication, Paris, Hachette, $3^{\mathrm{e}}$ édition. 
NDIAYE L. (2010), « La place du travail dans l'œuvre d’Ahmadou Bamba. Étude des représentations socioculturelles du travail en milieu mouride sénégalais », Revue sénégalaise de sociologie, vol. 7, $\mathrm{n}^{\circ}$ 10, p. 163-178.

NDIAYE M. (2013), « La presse en ligne au Sénégal. Nécessité économique ou sentinelle démocratique ?", in M.-C. Diop (dir.), Le Sénégal sous Abdoulaye Wade : le sopi à l'épreuve du pouvoir, Paris, CRES-Karthala, p. 689-706.

OueLLEt B. (2007), « Médias et religion à l'ère du zap et du clic : l'impact de la révolution numérique ", Médias et religion: un risque ou une chance ? L'impact des médias modernes sur l'expérience religieuse et la conscience sociale, Congrès mondial de l'Union catholique internationale de la presse, Université Bishop's, Sherbrooke (Canada), 3-10 juin.

Ross E. (2006), Sufi City: Urban Design and Archetypes in Touba, Rochester, University of Rochester Press.

SyLlA S. (2015), « Implications des médias confessionnels dans la vie religieuse et socio-politique. La communication à l'épreuve de la mission de service public sénégalais », Notes scientifiques. Homme et Société, n³, p. 43-65.

SyLLA S. (2017a), « De l'art de la propagande à l'art du management : Fonctions de la communication religieuse ", Revue Internationale des Francophonies, $\mathrm{n}^{\circ} 9$.

Sylla S. (2017b), Champ médiatique et pouvoir religieux : les ambiguités d'une relation au concours de l'avènement des médias confessionnels sénégalais, thèse de doctorat de sociologie, Saint-Louis du Sénégal, Université Gaston Berger.

THIAm E. H. I. S. (2010), Les aspects du Mouridisme au Sénégal, thèse de doctorat en sciences politiques, Siegen, Université de Siegen.

Willaime J.-P. (2001), « Les médias et les mutations contemporaines du religieux », Autres Temps. Cahiers d'éthique sociale et politique, vol. 69, nº 1, p. 64-75.

Wittmann F. (2008), « Politics, Religion and the Media: The Transformation of the Public Sphere in Senegal », Media, Culture e Society, vol. 30, n 4, p. 479-494. 